\title{
Relaxation strength of localized motions in D-sorbitol and mimicry of glass-softening thermodynamics
}

\author{
G. Power \\ Institute of Advanced Materials, Department of Electronic and Electrical Engineering, Trinity College, \\ Dublin 2, Ireland \\ G. P. Johari \\ Department of Material Science and Engineering, McMaster University, Hamilton, Ontario, \\ L8S 4L7, Canada \\ J. K. Vij ${ }^{\text {a) }}$ \\ Institute of Advanced Materials, Department of Electronic and Electrical Engineering, Trinity College, \\ Dublin 2, Ireland
}

(Received 19 February 2003; accepted 2 April 2003)

\begin{abstract}
The dielectric relaxation strength, $\Delta \epsilon_{\mathrm{JG}}$, the relaxation rate, $f_{m, \mathrm{JG}}$ and the distribution parameter, $\alpha_{\mathrm{JG}}$, of the faster relaxation process in D-sorbitol have been studied as a function of temperature and the cooling rate. Amongst these, $f_{m, \mathrm{JG}}$ and $\alpha_{\mathrm{JG}}$ of the glass and the supercooled liquid change smoothly with the temperature, $T$, but $\Delta \epsilon_{\mathrm{JG}}$ of the glassy state increases slowly on heating until the glass-softening range is reached and thereafter it increases rapidly at $T$ above the glass-softening temperature, $T_{g}$. Thus its plot against $T$ has an elbow-shape, remarkably similar to that observed for the volume, enthalpy and entropy. The derivative $\left(d \Delta \epsilon_{\mathrm{JG}} / d T\right)$ increases relatively abruptly at $T_{g}$ like the thermal expansion coefficient and the heat capacity of a glass. Thus $\Delta \epsilon_{\mathrm{JG}}$ is a function of the state's entropy and volume. The distribution of relaxation times became narrower as $T$ was increased, and $f_{m, \mathrm{JG}}$ increased according to the Arrhenius equation, $f_{m, \mathrm{JG}}=2.992 \times 10^{14} \mathrm{exp}$ $\left[-5.312 \times 10^{4} / R T\right]$, where $R=8.314 \mathrm{~J}(\mathrm{~K} \mathrm{~mol})^{-1}$. It is deduced that $f_{m, \mathrm{JG}}$ increases on structural relaxation of D-sorbitol. The results indicate that the relaxation mechanism involves motions of segments of the D-sorbitol molecules or of the whole molecule in local regions. (C) 2003 American Institute of Physics. [DOI: 10.1063/1.1577321]
\end{abstract}

\section{INTRODUCTION}

It is now recognized that in a low viscosity liquid there is only one dielectric relaxation process, with a narrow distribution of times. As the liquid is cooled, ${ }^{1}$ and its viscosity and density increase, a new relaxation, called the $\alpha$-process, develops. Similar development of the $\alpha$-process occurs when a liquid is polymerized and its viscosity and density increase at a fixed temperature. ${ }^{2,3}$ The strength of the $\alpha$-relaxation process grows rapidly at the expense of the strength of the original relaxation, and its rate rapidly decreases on cooling, ${ }^{1}$ and on further polymerization occurring isothermally. ${ }^{2,3}$ When this rate becomes comparable to one's measurements time scale, the liquid is said to have vitrified. In the vitrified state, the highly attenuated relaxation had been known as the secondary, or $\beta$-relaxation of a glass. To distinguish it from the $\beta$-relaxation process of the mode-coupling theory, the relaxation observed in glasses and highly viscous liquids has been called the Johari-Goldstein relaxation. ${ }^{4-6}$ Thus it seems that the relaxation process with a relatively narrow distribution of relaxation times in the low-viscosity liquid becomes two relaxation processes in a highly viscous liquid, each process with a broad distribution of relaxation times. Thus the Johari-Goldstein relaxation is not a characteristic of the

\footnotetext{
a) Author to whom correspondence should be addressed. Electronic mail: jvij@tcd.ie
}

glassy state, but rather that of a liquid. ${ }^{7}$ This is particularly evident in the study of D-sorbitol (Figs. 1 and 3 in Ref. 8), whose sub- $T_{g}$ dielectric relaxation strength has been found to linearly extrapolate to the dielectric relaxation strength of the only one process observed in the high temperature, low viscosity liquid.

There is a considerable interest in understanding the mechanism of the Johari-Goldstein relaxation for reasons mentioned in an earlier review. ${ }^{9}$ Since that review was written, it has been found that, (i) the dielectric relaxation strength of this process increases with increase in $T$ more rapidly at $T>T_{g}$ than at $T<T_{g},{ }^{10}$ (ii) the strength irreversibly decreases asymptotically with time like the enthalpy and volume of a rapidly quenched glass, ${ }^{11}$ (iii) both the rate of the relaxation and the dielectric strength are unchanged when electrolytes are dissolved in the liquid, ${ }^{12}$ and (iv) the relaxation process itself has been related to the low-energy excitations (the so-called Boson peak, or Poley absorption), in as much as it is due to molecular motions in regions of relatively low local density in a glass and crystal. ${ }^{13,14} \mathrm{We}$ also point out that in addition to the description provided in Ref. 9 , amorphous metal alloys, ${ }^{15}$ in which only translational motions of atoms are possible (atoms do not rotate), have been found to show the Johari-Goldstein relaxation in their mechanical relaxation, like molecular glasses and polymers. In the above-given aspects (i) and (ii), the dielectric strength of 
this relaxation mimics the characteristic temperature and time dependence of the thermodynamic properties of a glass.

The dielectric relaxation strength of this relaxation, $\Delta \epsilon_{\mathrm{JG}}$, is determined by the number of molecules capable of reorienting in a local region of a glass' or viscous liquid's structure, and its relaxation rate decreases with decrease in $T$ according to the Arrhenius equation. ${ }^{1,5,7,9,11,16-19}$ In several earlier studies, ${ }^{20-23}$ the $\Delta \epsilon_{\mathrm{JG}}$ against $T$ plots appear to have an elbowlike shape, and a thorough study ${ }^{10}$ of $\Delta \epsilon_{\mathrm{JG}}$ of a rigid molecular chlorobenzene-decalin mixture has shown that the slope change at this elbow-shape plot occurs at $T \approx T_{g}$. Moreover, a study of quenched 5-methyl-2-hexanol glass ${ }^{11}$ showed that on heating, $\Delta \epsilon_{\mathrm{JG}}$ first decreases irreversibly and then increases, thus producing a broad local minimum. A remarkable conclusion was that changes in $\Delta \epsilon_{\mathrm{JG}}$ observed with time and temperature of a glass mimic the changes in its enthalpy entropy and volume. Therefore, kinetic unfreezing of the density fluctuations which raise $C_{p}$ of a glass also raise its $\left(d \Delta \epsilon_{\mathrm{JG}} / d T\right)$.

Because of the significance of $\Delta \epsilon_{\mathrm{JG}}$ 's mimicry of the glass-softening thermodynamics, ${ }^{10,11}$ it is necessary to determine whether both the $C_{p}$-like increase in $\left(d \Delta \epsilon_{\mathrm{JG}} / d T\right)$ in the $T_{g}$ range and the broad minimum in $\Delta \epsilon_{\mathrm{JG}}$ can be observed in a single substance. For this purpose, a liquid with a relatively high magnitude of dipole moment was needed, and a search showed that D-sorbitol (D-glucitol, $\mathrm{C}_{6} \mathrm{H}_{8}(\mathrm{OH})_{6}$ ) has a dipole moment of ca. 3.3 Debye. Its liquid state has a hydrogen-bonded network structure, more complex than that of liquid glycerol. Dielectric and other relaxations of its supercooled liquid and glassy states have been studied by several groups ${ }^{8,20,22,24-37}$ (see also review in Ref. 11), and in some cases with conflicting results ${ }^{8,33}$ and unusual relaxation features. ${ }^{26}$ [Briefly, in Fig. 2 of Ref. 26, the Johari-Goldstein relaxation peak frequency shows a maximum at ca. $262 \mathrm{~K}$ in its plot against the temperature, instead of the usual monotonic increase with increase in the temperature. This means that there are two temperatures at which the relaxation rate is the same. We discuss this feature in relation to other studies in Sec. IV(ii).] The relaxation mechanism of this process has also been studied by NMR, ${ }^{38}$ and its relation to the theory of supercooled liquids has been examined. ${ }^{39}$ In our preliminary work, we accidentally discovered that sticky, liquid D-sorbitol left at ambient temperature for ca. 10 days became hard and brittle, indicating that it had crystallized. A literature search thereafter revealed that D-sorbitol has two crystalline forms, as indicated by calorimetry and $\mathrm{x}$-ray diffraction. ${ }^{40}$ Since some of the unusual findings in an earlier study may have been caused by crystallization, it seemed necessary also to study the $\epsilon^{\prime}$ and $\epsilon^{\prime \prime}$ spectra of D-sorbitol by avoiding its crystallization and by repeated measurements. Here we report a detailed study of the Johari-Goldstein relaxation in D-sorbitol glass obtained by cooling at different rates, and of the change in its characteristics with time and with temperature.

\section{EXPERIMENTAL METHODS}

The crystalline form of D-sorbitol (99.5\%) was purchased from Fluka AG and used without further treatment. Its dilatometric glass-softening temperature $T_{g}$ is known to be $265.3 \pm 1.5 \mathrm{~K}^{16,25}$ Its dielectric relaxation time has been found to be $100 \mathrm{~s}$ at $268 \mathrm{~K},{ }^{22}$ which has also been called $T_{g}$. The dielectric cell used was a miniature parallel plate condenser with 18 plates and a nominal capacitance of $27 \mathrm{pF}$. This capacitance was measured accurately in air prior to performing these experiments. A glass vial, $10 \mathrm{~mm}$ diameter and $33 \mathrm{~mm}$ length, was used to contain the liquid sample, in which the condenser was immersed. A small $100 \Omega$ Pt resistance sensor was used for monitoring the temperature. The temperature of the sample holder inside the cryostat, which was purchased from Oxford Instruments, Oxford, UK, was controlled by using an electronic temperature controller. The dielectric spectra of permittivity, $\epsilon^{\prime}$, and loss, $\epsilon^{\prime \prime}$, were continuously measured in the frequency domain by means of a Solartron FRA-1255A frequency response analyzer connected to a Chelsea dielectric interface.

For dielectric measurements, samples of D-sorbitol were prepared by heating its crystals at $400 \mathrm{~K}$ for $2-3 \mathrm{~h}$ in a separate glass container until its melt became transparent. The glass vial sample holder was filled with molten D-sorbitol, the capacitor was inserted in the vial and the assembly was transferred to a cryostat maintained at a temperature slightly above $390 \mathrm{~K}$. Once the temperature of the liquid had stabilized above $390 \mathrm{~K}$, the sample contained in the dielectric cell was thermally treated in five different ways, and five different metastable states of D-sorbitol were studied. For the first among these, referred to here as state (i), D-sorbitol was vitrified by cooling in the cryostat from 391.8 $\mathrm{K}$ to $221.1 \mathrm{~K}$ at a rate of $\sim 10 \mathrm{~K} \mathrm{~min}^{-1}$. It was then heated at $0.1 \mathrm{~K} \mathrm{~min}^{-1}$ to $271.2 \mathrm{~K}$ during which its $\epsilon^{\prime}$ and $\epsilon^{\prime \prime}$ spectra were measured at $0.33 \mathrm{~K}$ temperature intervals. For the second, named state (ii), D-sorbitol was quenched from $390.6 \mathrm{~K}$ to $77 \mathrm{~K}$ by immersing in liquid nitrogen and placed in the cryostat where it was heated rapidly to $220.4 \mathrm{~K}$. The temperature was then slowly increased to $281.0 \mathrm{~K}$ at 0.1 $\mathrm{K} \mathrm{min}{ }^{-1}$ during which its $\epsilon^{\prime}$ and $\epsilon^{\prime \prime}$ spectra were measured at $0.33 \mathrm{~K}$ temperature intervals. For the third, named state (iii), D-sorbitol was cooled from $392.9 \mathrm{~K}$ to $281.3 \mathrm{~K}$ at $\sim 5$ $\mathrm{K} \mathrm{min}^{-1}$, and thereafter slowly cooled to $221.6 \mathrm{~K}$ at 0.1 $\mathrm{K} \mathrm{min}^{-1}$, while its spectra were measured at $0.33 \mathrm{~K}$ intervals. For the fourth, named state (iv) D-sorbitol melt was quenched from $399.3 \mathrm{~K}$ to $77 \mathrm{~K}$ by immersing in liquid nitrogen and then heating rapidly to $205.3 \mathrm{~K}$. Its spectra were then measured at $T$ from $205.3 \mathrm{~K}$ to $234.3 \mathrm{~K}$ during heating at a $0.1 \mathrm{~K} / \mathrm{min}$ rate.

The mean time taken to measure the spectra in the frequency range $10 \mathrm{~Hz}-1 \mathrm{MHz}$ for samples (i)-(iii) was $\sim 206$ s. Since the spectra were obtained during the course of heating the sample at $0.1 \mathrm{~K} / \mathrm{min}$, the sample's temperature would have increased by $\sim 0.34 \mathrm{~K}$ in the course of these measurements. For the dielectric measurements on sample (iv), this time was only $\sim 186 \mathrm{~s}$ due to the narrower frequency range $(1 \mathrm{~Hz}-10 \mathrm{kHz})$ and the temperature rose by only $0.31 \mathrm{~K}$. A rise in temperature by $0.3 \mathrm{~K}$ would correspond to a maximum increase in the frequency of maximum dielectric loss, $f_{m}$, by approximately 0.09 decades (estimated by using the activation energy term given in Ref. 26), whereas for most measurements in the given frequency range, the increase in $f_{m}$ would be much lower than this value, and any change in 


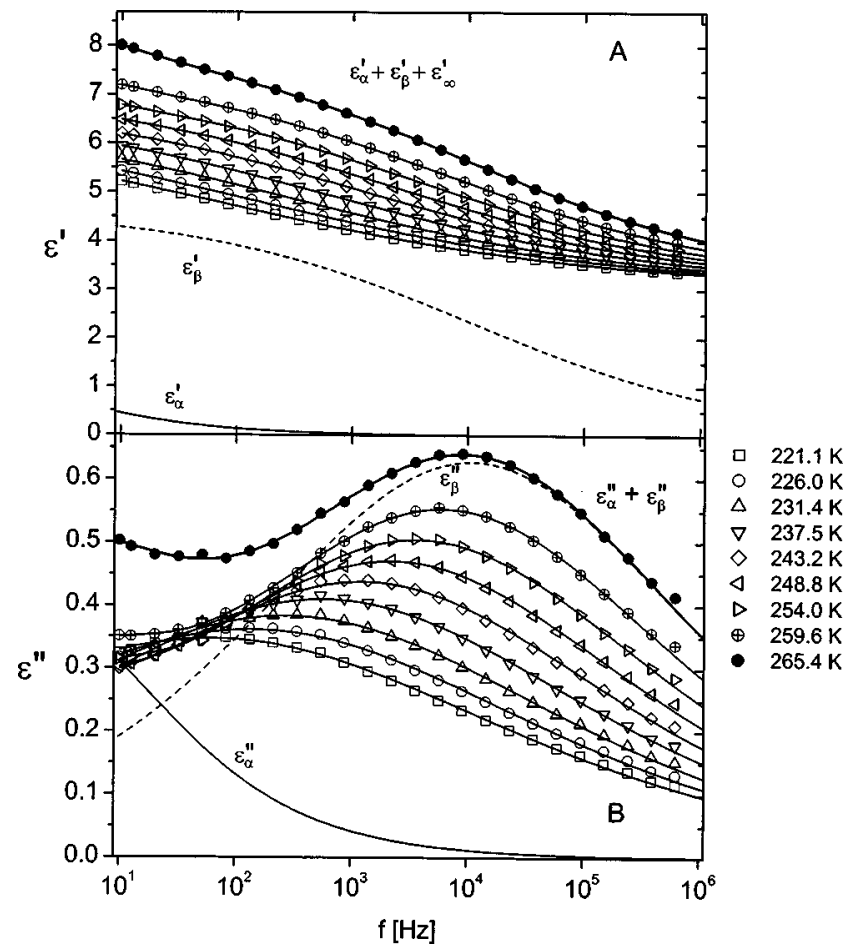

FIG. 1. (A) The $\epsilon^{\prime}$ spectra of the glassy state of D-sorbitol state (i) measured at different temperatures. The molten sample was first heated to $391.8 \mathrm{~K}$ and then cooled to $221.1 \mathrm{~K}$ at a rate of $\sim 10 \mathrm{~K} \mathrm{~min}^{-1}$. It was then heated to 271.2 $\mathrm{K}$ at $0.1 \mathrm{~K} \mathrm{~min}^{-1}$, and spectra were obtained continuously during this period. (B) The corresponding $\epsilon^{\prime \prime}$ spectra. The temperatures for the spectra are as indicated. In both panels the fitting of Eq. (1) is shown at one temperature, $265.4 \mathrm{~K}$. Curves labeled $\epsilon_{\alpha}^{\prime}, \epsilon_{\beta}^{\prime}, \epsilon_{\alpha}^{\prime \prime}$, and $\epsilon_{\beta}^{\prime \prime}$ and their respective sums were obtained by fitting the data. The parameters used for the fit are $\Delta \epsilon_{\alpha}$ $=1.62, \tau_{\alpha}=6.77 \times 10^{-2} \mathrm{~s}, \alpha_{\alpha}=0.549, \beta_{\alpha}=1.00, \Delta \epsilon_{\mathrm{JG}}=4.64, \tau_{\mathrm{JG}}=1.49$ $\times 10^{-5} \mathrm{~s}, \alpha_{\mathrm{JG}}=0.336$, and $\beta_{\mathrm{JG}}=1.00$.

the broadening parameters would also be negligibly small. This may also have a slight effect on the shape of the spectra because measurements at one extreme of frequency are performed at a temperature $\sim 0.3 \mathrm{~K}$ less than measurements on the other extreme of frequency. Considering the broadness of the spectra and the negligible change in $f_{m}$ the effect of this temperature uncertainty is neglected here. The temperature given here for each $\epsilon^{\prime}$ and $\epsilon^{\prime \prime}$ spectrum is the average of 10 temperature readings measured during the frequency scan.

\section{RESULTS}

The $\epsilon^{\prime}$ and $\epsilon^{\prime \prime}$ spectra of state (i) are shown at several temperatures in Figs. 1(A) and 1(B). The general form of the spectra is a broad relaxation peak and low frequency "wing" in $\epsilon^{\prime \prime}$, and a step in $\epsilon^{\prime}$. It is seen that as $T$ is increased, the relaxation peak becomes narrower, its height increases and the peak bodily shifts to higher frequencies. It is possible to analyze the $\epsilon^{\prime}$ and $\epsilon^{\prime \prime}$ spectra to obtain useful parameters in several ways. The method adopted here and used by the authors ${ }^{10-12,17}$ and others, ${ }^{22,41}$ is to fit a superposition of Havriliak-Negami empirical equation. ${ }^{42}$ For fitting of the equation to the data on D-sorbitol liquid and glass, two relaxation processes were used, one set of terms for the $\alpha$-process and the second set for the $\beta$-process. Thus, the equation for the frequency-dependent complex permittivity, $\epsilon^{*}(\omega)$, has the form

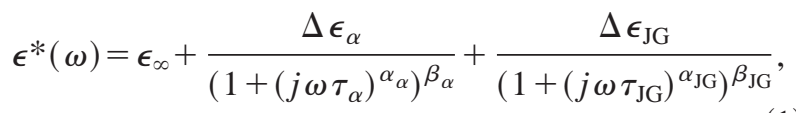

where $\omega$ is the angular frequency, $\epsilon_{\infty}$ is the high frequency dielectric permittivity, $\Delta \epsilon_{\alpha}$ is the dielectric relaxation strength of the $\alpha$-process, $\tau_{\alpha}$ is the relaxation time of the $\alpha$-process, and $\alpha_{\alpha}$ and $\beta_{\alpha}$ are the Cole-Cole and ColeDavidson parameters ${ }^{43}$ describing, respectively, the symmetric and asymmetric broadening of the $\alpha$-relaxation process with respect to a Debye-type relaxation for which $\alpha=\beta=1$. The parameters, $\Delta \epsilon_{\mathrm{JG}}, \tau_{\mathrm{JG}}, \alpha_{\mathrm{JG}}$, and $\beta_{\mathrm{JG}}$ are the analogous quantities for the $\beta$-relaxation process. As only the high frequency portion of the emerging $\alpha$-process appears in the frequency and temperature range of these experiments, values of the parameters for the $\beta$-process are reliably obtained. Since there is insignificant or no contribution from dc conductivity at these temperatures, its effect on $\epsilon^{\prime}$ and $\epsilon^{\prime \prime}$ has been neglected.

The frequency of maximum loss, $f_{m, i}$, of a relaxation process $i$ is used to quantify its rate. It may be determined using the equation, ${ }^{41}$

$$
f_{m, i}=\left(2 \pi \tau_{i}\right)^{-1}\left[\sin \left(\frac{\alpha_{i} \pi}{2+2 \beta_{i}}\right)\right]^{1 / \alpha_{i}}\left[\sin \left(\frac{\alpha_{i} \beta_{i} \pi}{2+2 \beta_{i}}\right)\right]^{-1 / \alpha_{i}},
$$

where $\tau_{i}$ is the relaxation time, and $\alpha_{i}$ and $\beta_{i}$ are the set of broadening parameters for the $i$ th process ( $\alpha$-process, or the Johari-Goldstein process) and $f_{m, i}$ the frequency of its $\epsilon^{\prime \prime}$ peak, as indicated in Eq. (1). This equation was applied to the fitting parameters of the $\beta$-process to obtain values of $f_{m, \mathrm{JG}}$. The contributions to $\epsilon^{\prime}$ and $\epsilon^{\prime \prime}$ from the $\alpha$-relaxation process at the low-frequency side of the spectra were deconvoluted from the contributions to $\epsilon^{\prime}$ and $\epsilon^{\prime \prime}$ from the Johari-Goldstein relaxation process. One such deconvolution of the spectra at $265.4 \mathrm{~K}$ is shown in Figs. 1(A) and 1(B). The $\epsilon^{\prime}$ and $\epsilon^{\prime \prime}$ spectra of the respective processes are shown by continuous and dashed lines, as labeled. The fitting was found to be best when the Cole-Davidson $\beta$-parameter for the Johari-Goldstein process, $\beta_{\mathrm{JG}}$, was equal to 1.00 at all temperatures and all samples of liquid and glassy D-sorbitol in their different states (i)-(iv).

There are three characteristics of a relaxation process: (i) the strength of relaxation, (ii) the distribution of relaxation rates, which appears as its spectral half-width of more than 1.14 decades and is usually discussed in terms of distribution parameters, $\alpha$ and $\beta$, and, (iii) the relaxation rate. The plots of the strength of relaxation, $\Delta \epsilon_{\mathrm{JG}}$, against $T$ for D-sorbitol's states (i)-(iii) are shown in Fig. 2(A). As observed for the chlorobenzene-decalin mixture, ${ }^{10} \Delta \epsilon_{\mathrm{JG}}$ of states (i) and (iii) increases slowly initially and, then as the glass softens, it increases rapidly. For D-sorbitol, this rapid increase begins at $\sim 265 \mathrm{~K}$. In contrast, Nozaki, et al. ${ }^{8}$ have found that its $\Delta \epsilon_{\mathrm{JG}}$ increased smoothly from $270 \mathrm{~K}$ to $395 \mathrm{~K}$, and at $T$ $<270 \mathrm{~K}$, the $\Delta \epsilon_{\mathrm{JG}}$ values were scattered (Fig. 3 in Ref. 8). A change similar to that seen in Fig. 2(A) had been observed 

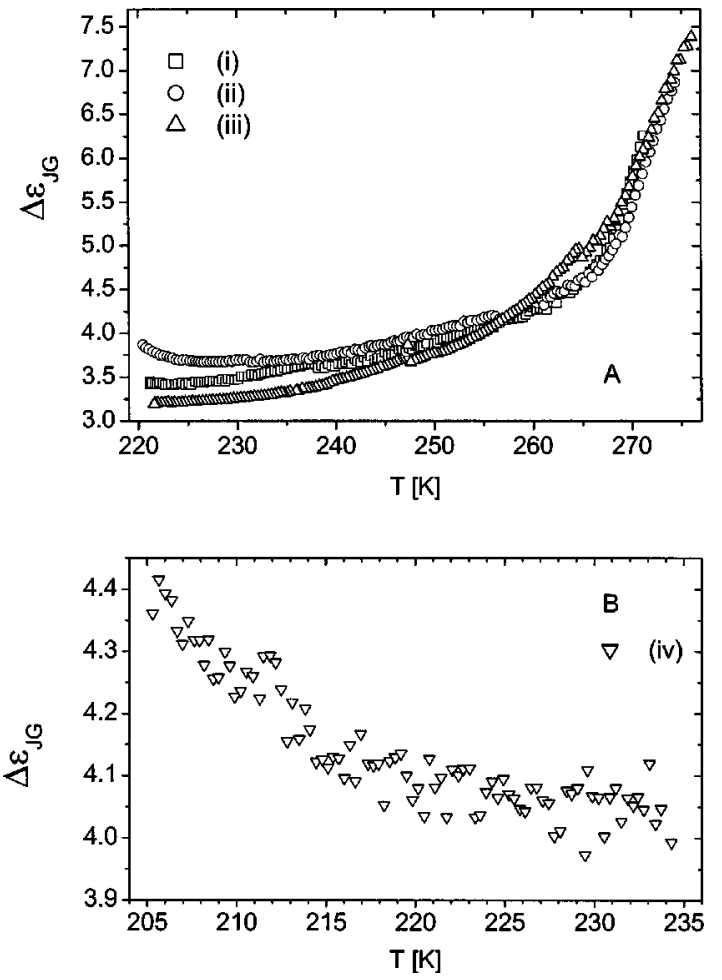

FIG. 2. (A) The dielectric relaxation strength, $\Delta \epsilon_{\mathrm{JG}}$, of the glassy and liquid states of D-sorbitol is plotted against the temperature. Notations used for states (i)-(iii) are as indicated. (B) The $\Delta \epsilon_{\mathrm{JG}}$, of glassy D-sorbitol state (iv), which was obtained by quenching in liquid nitrogen, is plotted against the temperature.

for D-sorbitol, ${ }^{20,22}$ and other liquids, ${ }^{10,18,23}$ as well as for a polymer, ${ }^{21}$ but was not considered in terms of the variation of the enthalpy, $H$, entropy, $S$, and volume, $V$, with the temperature. In Fig. 2(A), $\Delta \epsilon_{\mathrm{JG}}$ of state (ii) decreases on heating from $220 \mathrm{~K}$ to $230 \mathrm{~K}$, showing a shallow minimum before $\Delta \epsilon_{\mathrm{JG}}$ begins to increase. This is similar to the feature observed for 5-methyl-2-hexanol, for which the minimum was more pronounced. ${ }^{11}$ The value of $\Delta \epsilon_{\mathrm{JG}}$ of D-sorbitol's state (iv) is plotted against $T$ in Fig. 2(B). It decreases with increase in $T$ and appears to reach a broad local minimum.

The distributions of relaxation times parameter, $\alpha_{\mathrm{JG}}$ of the states (i)-(iv) is plotted against $T$ in Fig. 3(A). For states (i)-(iv), $\alpha_{\mathrm{JG}}$ increases on heating. Data for states (i) and (ii) agree within the errors, those for state (iii) are slightly higher at low temperatures, while those for state (iv) are ca. $4.1 \%$ higher than that of state (i) at 221.1 K. For all states (i)-(iv), $\alpha_{\mathrm{JG}}$ increases with increase in $T$, from 0.253 at $221.1 \mathrm{~K}$ to 0.346 at $271.2 \mathrm{~K}$ for state (i), from 0.247 at $220.4 \mathrm{~K}$ to 0.351 at $274.4 \mathrm{~K}$ for state (ii), from 0.256 at 221.6 to 0.371 at 276.1 $\mathrm{K}$ for state (iii), and from 0.232 at $205.3 \mathrm{~K}$ to 0.299 at 234.3 $\mathrm{K}$ for state (iv).

The plots of $f_{m, \mathrm{JG}}$ against $T$ are shown in Fig. 3(B). The plots for samples (i)-(iv) lie close to each other, showing that the sample history has a relatively small effect on $f_{m, \mathrm{JG}}$. This sample history dependence of $f_{m, \mathrm{JG}}$ is consistent with the effect of density increase observed by Naoki and Ujita. ${ }^{16}$ They had found that $f_{m, \mathrm{JG}}$ of D-sorbitol vitrified under a pressure of 785 bar and studied at 1 bar was $23 \%$ higher than $f_{m, \mathrm{JG}}$ of the sample that had been vitrified at 1 bar by cooling

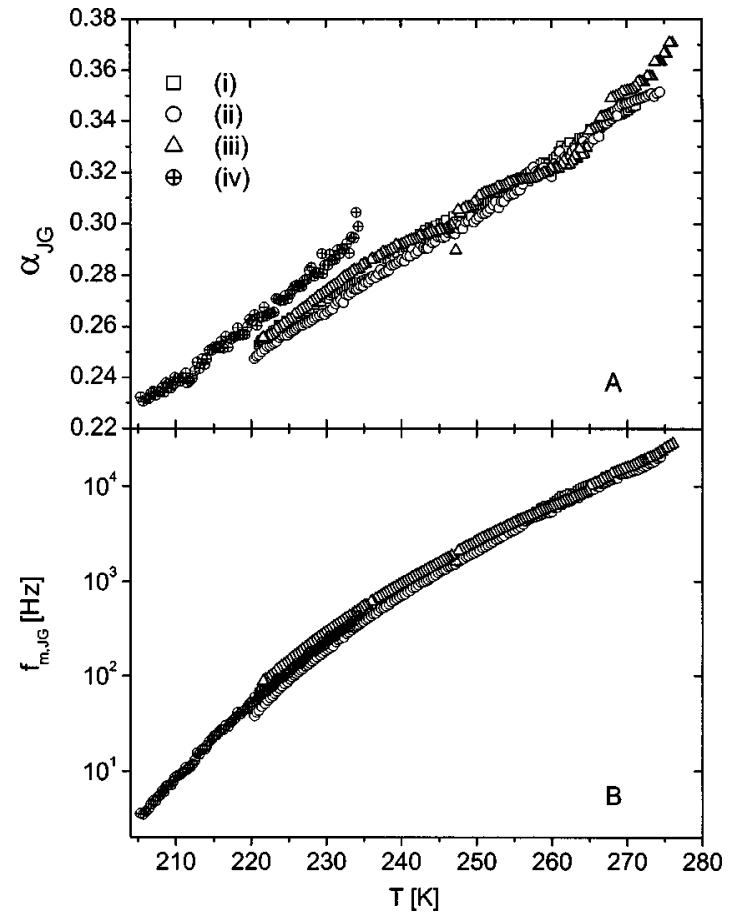

FIG. 3. (A) The distribution parameter, $\alpha_{\mathrm{JG}}$, for the glassy state relaxation of D-sorbitol is plotted against the temperature. Notations for states, (i)-(iv) are indicated. (B). The peak frequency, $f_{m, \mathrm{JG}}$, of the $\epsilon^{\prime \prime}$ spectra of glassy D-sorbitol is plotted against the temperature for states (i)-(iv), with different thermal histories, as described in the text.

at $0.2 \mathrm{~K} / \mathrm{min}$ and at $2.0 \mathrm{~K} / \mathrm{min}$ rates. However, Naoki and Ujita, ${ }^{16}$ had also found that several features of the JohariGoldstein relaxation in D-sorbitol are distinguished from those of molecular and polymeric glasses. In particular, (i) $\Delta \epsilon_{\mathrm{JG}}$ of a $0.6 \%$ higher density D-sorbitol glass obtained by vitrifying under 785 bar pressure was found to be larger and the distribution of relaxation times narrower than those of the glass obtained by vitrifying at 1 bar pressure, (ii) $\Delta \epsilon_{\mathrm{JG}}$ of both the higher and lower density glasses was found to increase slightly with increase in pressure. ${ }^{16}$ Similar effects have been observed in ion-containing alcohols and attributed to the hydrogen-bond breaking effects and electrostriction. ${ }^{44}$

However, Nozaki et al. ${ }^{8}$ also reported that $\epsilon_{s}$ of liquid D-sorbitol decreases with decrease in $T$, and $\Delta \epsilon_{\mathrm{JG}}$ increases, which means that contribution to $\Delta \epsilon$ from the $\alpha$-relaxation process decreases with decrease in $T$. In contrast, more recent studies have shown that $\Delta \epsilon$ of the $\alpha$-relaxation process increases with decrease in $T$ (Fig. 7 in Ref. 22). Since such inconsistencies can also arise if samples of D-sorbitol used by different groups contained different impurities and/or their respective data analysis differed, we do not discuss the observations on $\Delta \epsilon_{\mathrm{JG}}$ in relation to $\epsilon_{s}$.

\section{DISCUSSION}

\section{A. Resemblance with the glass-softening thermodynamics}

A general discussion of the origin of the JohariGoldstein relaxation and the effects of structural relaxation and temperature and pressure on it has been provided already, 9,11 as have its entropic consequences and its impli- 
cations for the potential energy landscape picture. ${ }^{9} \mathrm{Ngai}^{45}$ has reviewed experimental aspects of this subject in relation to coupling model. Therefore, we discuss here only the relevance of our observations for D-sorbitol to the general findings of this relaxation process, and interpret the differences observed.

The elbow-shape plot of $\Delta \epsilon_{\mathrm{JG}}$ against $T$ in Fig. 2(A) is similar to that observed for D-sorbitol, ${ }^{20,22}$ tetramethylbisphenol-A polycarbonate, ${ }^{21}$ toluene ${ }^{23}$ and a chlorobenzene-decalin mixture. ${ }^{10}$ Here, an additional small decrease in $\Delta \epsilon_{\mathrm{JG}}$ is observed also for state (ii) at $220-230 \mathrm{~K}$ in Fig. 2(A), and the plots of states (i)-(iii) do not superpose at low temperatures. The differences tend to become systematically less and vanish at $T>270 \mathrm{~K}$. Since $T_{g}$ of D-sorbitol as characterized by dilatometry is $265.3 \pm 1.5 \mathrm{~K}^{16,25}$ and by its dielectric relaxation rate of $100 \mathrm{~s}$ is $268 \mathrm{~K},{ }^{20,22}$ the difference vanishes in the equilibrium state of the liquid at $270 \mathrm{~K}$. The lack of superposition of the curves indicates that thermal history of the sample, as identified by states (i)-(iv) does have a small effect on $\Delta \epsilon_{\mathrm{JG}}$. As discussed earlier, ${ }^{10,11}$ the shape of these plots is remarkably similar to the shape of the plots of $V, H$, and $S$ against $T$ obtained on heating of a glass to $T>T_{g}{ }^{46}$

In Fig. 2(B), the magnitude of $\Delta \epsilon_{\mathrm{JG}}$ decreases irreversibly on heating a quenched sample from $205 \mathrm{~K}$. This effect was first observed in a study of 5-methyl-2-hexanol. ${ }^{11}$ As a result, there was a broad and shallow local minimum at ca. $145 \mathrm{~K}$, and thereafter a rise in $\Delta \epsilon_{\mathrm{JG}}$ smoothly occurring through its $T_{g}$ of $148 \mathrm{~K}^{11}$ The irreversible decrease in $\Delta \epsilon_{\mathrm{JG}}$ with increase in $T$ is also remarkably similar to the irreversible decrease in $V, H$, and $S$ observed generally for glasses obtained by rapid cooling. ${ }^{46}$ Such an isothermally occurring decrease in $V, H$, and $S$ has been interpreted also in terms of an asymptotic decrease in the fictive temperature. ${ }^{46}$ Also when a glass obtained by rapidly cooling a liquid is heated at a fixed rate, its $V, H$, and $S$ first decrease and then increase to meet the liquid's equilibrium curve. These changes have been interpreted in terms of first a decrease in the fictive temperature and then an increase after the temperature of the equilibrium line has been crossed at a certain heating rate. ${ }^{46}$ The corresponding changes observed in $\Delta \epsilon_{\mathrm{JG}}$ in this study, which are referred to as mimicry of the thermodynamic behavior in this and earlier studies, ${ }^{10,11}$ have been discussed in more detail in connection with the broad minimum in $\Delta \epsilon_{\mathrm{JG}}$ observed in the study of 5-methy-2-hexanol glass in Ref. 11.

Starting from a vanishingly small value at low temperatures, the area under the $\epsilon^{\prime \prime}$ peak, which is a measure of $\Delta \epsilon_{\mathrm{JG}}$, had been found to increase reversibly on heating and cooling of amorphous polymers ${ }^{47}$ and molecular glasses, ${ }^{18}$ as long as the glass was kept at temperatures far below its $T_{g}$. An irreversible decrease in the area under the $\epsilon^{\prime \prime}$ peak after annealing and slowly cooling had been observed in earlier studies, ${ }^{1,7,18,47-51}$ but it was not observed on heating a glass, partly because the rapidly cooled state relaxed during the 30 minutes of manually collecting the data at different frequencies, in contrast to ca. 3-4 min here, and partly because the data itself were less precise.

\section{B. Time and temperature effects on relaxation characteristics}

It is a general feature of the glassy state that its structure relaxes spontaneously with time. This appears as a spontaneous decrease in $V, H$, and $S$ towards the magnitudes of the corresponding values for the equilibrium liquid. It has been recently shown that $C_{p}$ of a glass also decreases spontaneously $y^{52}$ during the structural relaxation. On heating, the magnitude of these properties increases. When these properties are measured during the heating of a rapidly cooled glass whose structure relaxes, the magnitude of $V, H$, and $S$ decreases with time and increases with $T$, according to the relation

$$
\frac{d \theta}{d T}=\left(\frac{\partial \theta}{\partial t}\right)_{T}\left(\frac{1}{q}\right)+\left(\frac{\partial \theta}{\partial T}\right)_{t},
$$

where $\theta$ denotes $V, H$, or $S$ of the glass, and $q(=d T / d t)$ is the heating rate. The term for a fixed $t$ here and henceforth refers to the condition when no structural relaxation occurs. In the equilibrium state of a liquid, $V, H$, or $S$ do not change with time and therefore the change is determined only by the second term on the right-hand side (RHS) of Eq. (3). In simple terms, $(\partial \theta / \partial t)_{T}$ is negative and approaches zero as $t$ increases, and $(\partial \theta / \partial T)_{t}$ is positive and increases as $T$ increases, and their combined effects produce a broad minimum in the plot of $\theta$ against $T$ (see Ref. 54 for details). When the heating rate, $q$, is low, the minimum is broad and shallow and appears at a lower $T$, and when $q$ is high, the minimum is narrow and deep and appears at a higher $T$. For a given heating rate, a rapidly cooled glass shows a more pronounced minimum than a slow-cooled, or rapidly cooled but preannealed, glass. ${ }^{53}$

Evidently, $\Delta \epsilon_{\mathrm{JG}}$ mimics the behavior observed for $V, H$, or $S$ according to Eq. (3). The decrease in $\Delta \epsilon_{\mathrm{JG}}$ on heating of D-sorbitol's states (ii) and (iv) is a combination of the structural relaxation and temperature. The shallow minimum observed in the plot of $\Delta \epsilon_{\mathrm{JG}}$ against $T$ in curve (ii) in Fig. 2(A) and the data points shown in Fig. 2(B) may be understood in terms of the time-, and temperature-dependent changes in $\Delta \epsilon_{\mathrm{JG}}$, as follows: The net change in $\Delta \epsilon_{\mathrm{JG}}$ of a glass is the sum of the changes due to a decrease in the structural relaxation rate with time, and an increase in $T$ :

$$
d \Delta \epsilon_{\mathrm{JG}}=\left(\frac{\partial \Delta \epsilon_{\mathrm{JG}}}{\partial t}\right)_{T} d t+\left(\frac{\partial \Delta \epsilon_{\mathrm{JG}}}{\partial T}\right)_{t} d T,
$$

where the first term on the RHS of Eq. (4) represents the effect of structural relaxation at a fixed $T$, and the second term the effect of the increase in $T$ at a formally fixed $t$. On dividing by $d T$,

$$
\frac{d \Delta \epsilon_{\mathrm{JG}}}{d T}=\left(\frac{\partial \Delta \epsilon_{\mathrm{JG}}}{\partial t}\right)_{T} \frac{1}{q}+\left(\frac{\partial \Delta \epsilon_{\mathrm{JG}}}{\partial T}\right)_{t}
$$

where $q(=d T / d t)$ is the heating rate used during the course of measurements of the spectra, which is $0.1 \mathrm{~K} / \mathrm{min}(=1.67$ $\mathrm{mK} / \mathrm{s})$. The quantity $\left(\partial \Delta \epsilon_{\mathrm{JG}} / \partial T\right)_{t}$, which represents change $\Delta \epsilon_{\mathrm{JG}}$ with $T$ when no structural relaxation occurs, is positive, ${ }^{7,8,10,11,16,20,22}$ and the quantity $\left(\partial \Delta \epsilon_{\mathrm{JG}} / \partial t\right)_{T}$ is negative. ${ }^{10,11}$ Thus the minimum in the plot for state (ii) and 
(iv) in Figs. 2(A) and 2(B) appears at a temperature when $q^{-1}\left(\partial \Delta \epsilon_{\mathrm{JG}} / \partial t\right)_{T}=-\left(\partial \Delta \epsilon_{\mathrm{JG}} / \partial T\right)_{t}$ in Eq. (5). This means that the minimum would appear at a lower $T$ if $q$ is chosen to be low, and at a higher $T$ when $q$ is chosen to be high. In our study, this condition is satisfied at $230 \pm 4 \mathrm{~K}$ for sample (ii), for which the minimum is very broad. At $T<226 \mathrm{~K}$, structural relaxation dominates the change in $\Delta \epsilon_{\mathrm{JG}}$ during the measurements on heating, and at $T>233 \mathrm{~K}$, the increase in $T$ dominates the change in $\Delta \epsilon_{\mathrm{JG}}$.

In the spontaneous structural relaxation of a glass, a relatively large number of molecules diffuse but each diffuses by a very small distance. The rate of the $\alpha$-relaxation process generally decreases on structural relaxation of a glass. ${ }^{46}$ On combining with the observation on decrease in $\Delta \epsilon_{\mathrm{JG}}$, this indicates that there is a relation between the slowing of $\alpha$-relaxation process on structural relaxation and the slowing of the decrease in $\Delta \epsilon_{\mathrm{JG}}$.

It is possible to estimate the rate of change of $\log \left(f_{m, \mathrm{JG}}\right)$ at the annealing temperature indirectly from the plots of $\log \left(f_{m, \mathrm{JG}}\right)$ against $T$ or against $1 / T$ as done earlier. ${ }^{11}$ The change in $f_{m, \mathrm{JG}}$ with $T$ may be written as a sum of timedependent and temperature-dependent changes as

$$
\frac{d \log \left(f_{m, \mathrm{JG}}\right)}{d T}=\left(\frac{\partial \log \left(f_{m, \mathrm{JG}}\right)}{\partial t}\right)_{T}\left(\frac{1}{q}\right)+\left(\frac{\partial \log \left(f_{m, \mathrm{JG}}\right)}{\partial T}\right)_{t}
$$

and the change with $1 / T$ is given by

$$
\begin{aligned}
\frac{d \log \left(f_{m, \mathrm{JG}}\right)}{d(1 / T)}= & \left(\frac{\partial \log \left(f_{m, \mathrm{JG}}\right)}{\partial t}\right)_{T}\left(\frac{d t}{d(1 / T)}\right) \\
& +\left(\frac{\partial \log \left(f_{m, \mathrm{JG}}\right)}{\partial(1 / T)}\right)_{t} .
\end{aligned}
$$

The first term on the RHS in Eqs. (6) and (7) represents the effect of structural relaxation and the second term the effect of thermal energy. Thus during measurements on a quenched sample with increasing $T$, the slope of the plot of $\log \left(f_{m, \mathrm{JG}}\right)$ against inverse temperature will be affected by the structural relaxation until a temperature is reached where the equilibrium state has been attained during the time of heating. Consequently, a change in the slope of the plot from the Arrhenius equation $\left[f_{m, \mathrm{JG}}=A \exp \left(-E^{*} / R T\right)\right.$, where $A$ is a constant, $E^{*}$ the Arrhenius energy and $R$ is the gas constant], would be a reflection of a time-dependence of $f_{m, \mathrm{JG}}$ during the course of the dielectric measurements, when the first term on the RHS in Eq. (7) is significant. The magnitude of $(1 / q)$ in the first term in RHS of Eq. (6) is fixed at $600 \mathrm{~s} \mathrm{~K}^{-1}$, and $\left[d t / d(1 / T)=\left(-T^{2} / q\right)\right]$ in the first term on RHS of Eq. (7) decreases from $-2.915 \times 10^{7} \mathrm{~s} \mathrm{~K}$ at $220.4 \mathrm{~K}$, to -4.518 $\times 10^{7} \mathrm{~s} \mathrm{~K}$ at $274.4 \mathrm{~K}$. Therefore, a very small rate of increase in $f_{m, \mathrm{JG}}$ on spontaneous relaxation at a fixed $T$ would be substantially increased in magnitude on multiplication by the large value of $600 \mathrm{~s} \mathrm{~K}^{-1}$ in Eq. (6) and of $10^{7} \mathrm{~s} \mathrm{~K}$ in Eq. (7).

An examination of the change in the slope of the plot in Figs. 3(B) and 4 shows that $f_{m, \mathrm{JG}}$ for state (ii), the quenched state, is lower than that of state (iii), the structurally relaxed state even thought the latter is macroscopically denser. This indicates that the term $\left[\partial \log \left(f_{m, \mathrm{JG}}\right) / \partial t\right]_{T}$ is positive. Since $[d t / d(1 / T)]$ is negative, the product of the two terms is

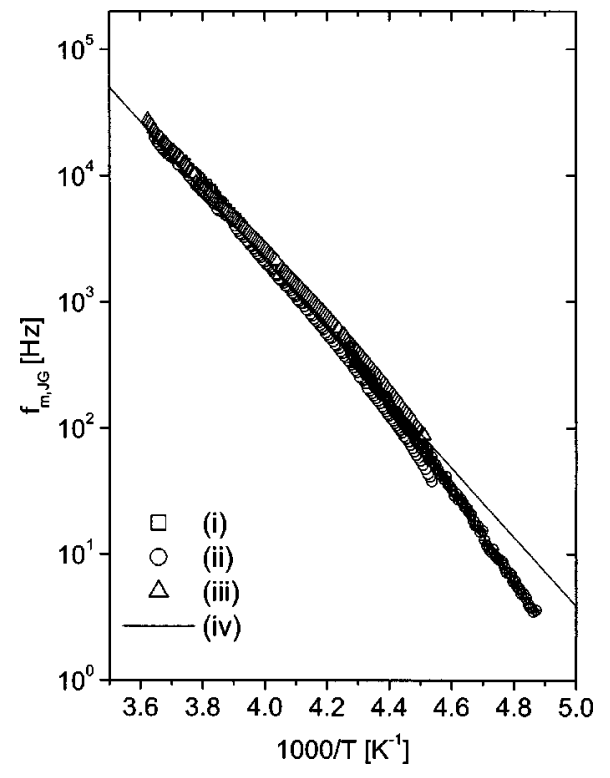

FIG. 4. The frequency at which the maximum of the dielectric loss peak, $f_{m, \mathrm{JG}}$, in glassy D-sorbitol appears is plotted logarithmically against the reciprocal temperature. The symbols used for the data corresponding to the various states are indicated. The solid line is a fit of the Arrhenius equation, $f_{m}=1.96 \times 10^{14} \exp \left(-6.31 \times 10^{3} / T\right)$ to the data of state (ii) at $T>250 \mathrm{~K}$.

negative, which adds to the negative second term in Eq. (7). The net result is that the slope of the Arrhenius plot is more negative when structural relaxation occurs during the measurements. To estimate its value at $221.1 \mathrm{~K}$ from the plot in Fig. 4, we extend the Arrhenius fit to the data from high temperatures to $221.1 \mathrm{~K}$ as shown by the continuous line. Knowing $[d t / d(1 / T)]$ from the heating rate, $\left[\partial \log \left(f_{m, \mathrm{JG}}\right) / \partial(1 / T)\right]_{t}$ from the slope of the extended line, and $\left[d \log \left(f_{m, \mathrm{JG}}\right) / d(1 / T)\right]$ from an estimate of the actual slope in Fig. 4 at $221.1 \mathrm{~K}$, we determine $\left[\partial \log \left(f_{m, \mathrm{JG}}\right) / \partial t\right]_{T}$ as $\sim 7.54 \times 10^{-5} \log (\mathrm{Hz}) / \mathrm{s}$. This means that $f_{m, \mathrm{JG}}$ would increase on structural relaxation. This is a significant deduction. Whether it is peculiar to the H-bonded network structure of D-sorbitol, or whether it is a general feature of the glassy state requires isothermal measurements on a variety of glasses. Such measurements are particularly necessary because the downwards bending of the $\log \left(f_{m, \mathrm{JG}}\right)$ against $1 / T$ plots has been observed for several glasses before, and attempts have been made to fit empirical equations to such curves in the same manner as for the $\alpha$-relaxation process observed in the equilibrium state of a liquid.

We now consider the results obtained for the temperature dependence of $\log \left(f_{m, \mathrm{JG}}\right)$ or the rate of the $\beta$-relaxation process in D-sorbitol by other groups. As mentioned in Sec. I, Olsen $^{26}$ has found that $\log \left(f_{m, \mathrm{JG}}\right)$ first increases with increase in $T$, reaches a maximum at ca. $262 \mathrm{~K}$, and thereafter decreases as $T$ is increased (Fig. 2, Ref. 26). This is contrary to the usual observation that the rate of a relaxation process increases with increase in the temperature, and does not decrease. Since our results in Fig. 4 differ from theirs, ${ }^{26}$ we searched in the literature if other studies of D-sorbitol agree with the finding of the maximum in $\log \left(f_{m, \mathrm{JG}}\right)$ against $T$ plot. Studies reported before 1998 and already cited here have not shown this maximum. Rather, these have shown an Arrhen- 
ius plot of $\log \left(f_{m, \mathrm{JG}}\right)$ against $1 / T$. In the studies published after 1998, we find that a slight decrease in slope followed by a rapid increase has been observed in the plot of $\log \left(f_{m, \mathrm{JG}}\right)$ vs $T$ in Fig. 7, Ref. 22 [i.e., the slope of the $\log \left(f_{m, \mathrm{JG}}\right)$ vs $1 / T$ plot becoming more negative at higher temperatures]. Fujima et al. have plotted the relaxation time at maximum loss, $\tau_{m, \mathrm{JG}}$, for the $\beta$-process in Fig. 3 of Ref. 29 , and shown that there is only an increase in slope in the plot of $\log \left(\tau_{m, \mathrm{JG}}\right)$ against $1 / T$ at higher temperatures. Nozaki et al. (Fig. 2 in Ref. 32) also found the same behavior within the experimental errors. It is also worth noting that, as in Olsen's ${ }^{26}$ earlier studies of D-sorbitol, a broader and less well-defined maximum in the $\log \left(f_{m, \mathrm{JG}}\right)$ against $1 / T$ plots was also reported by Olsen et al.,${ }^{54}$ for pyridine-toluene solution and tripropylene glycol (Fig. 2 in Ref. 54) samples cooled and heated during the course of measurements. They ${ }^{54}$ also noted that similar behavior was observed for two other liquids.

These results are puzzling, and their resolution seems difficult because experimental and data analysis details are not available, nor is it certain whether or not several samples, or repeated measurements, had shown the same results. Therefore, it is not certain whether such a maximum is an artifact of data analysis in a temperature region where the relaxation rates of the $\alpha$-process and the Johari-Goldstein relaxation approach each other and the relaxation strengths of the two become comparable, or whether it is an artifact of experimental procedure. In contrast, a variety of studies, intended to examine relaxation processes in this region, now possible with the accurate and computer-controlled dielectric measurements, have not shown such a maximum in the plots of $\log \left(f_{m, \mathrm{JG}}\right)$ against $T$ (or $1 / T$ ). The Fujima et al. study ${ }^{55}$ of salol performed in the $10 \mathrm{MHz}$ to $5 \mathrm{GHz}$ range also shows no such maximum in $\log \left(f_{m, \mathrm{JG}}\right)$. Rather it shows an Arrhenius behavior. A number of studies performed by Kudlik et al. (Figs. 9 and 10 in Ref. 23) also do not show such a maximum, or a highly decreased magnitude of the slope of the $\log \left(f_{m, \mathrm{JG}}\right)$ against $1 / T$ plots of several other liquids. However, the thermal history dependence of $\log \left(f_{m, \mathrm{JG}}\right)$ detected in earlier studies ${ }^{26,54}$ is substantiated here, but in a limited sense.

\section{Relaxation mechanism in D-sorbitol}

Since a detailed discussion on the origin of this relaxation has been provided already, ${ }^{9,11}$ only a brief description relevant to D-sorbitol is needed here. Solvation dynamics ${ }^{27}$ experiments, time resolved electric field relaxation ${ }^{31}$ and ${ }^{2} \mathrm{H}$ NMR studies of D-sorbitol ${ }^{35}$ have all been interpreted to mean that in the Johari-Goldstein process, essentially all molecules reorient by a small, nearly temperatureindependent angle. A similar conclusion has been deduced from its dielectric studies, ${ }^{22,34}$ and by the observed effects of crystallization on its $\Delta \epsilon_{\mathrm{JG}} \cdot{ }^{33}$ But since the strength of a relaxation process to which a temperature-independent (angular) reorientation of all molecules contributes is expected to vary inversely with $T$, this picture presents a difficulty in reconciling the observed opposite effect in Fig. 2(A) here, and also reported elsewhere, ${ }^{20,22}$ i.e., $\Delta \epsilon_{\mathrm{JG}}$ of D-sorbitol liquid increases reversibly with increase in $T$.
Moreover, as structural relaxation of a glass brings its state to higher density (or lower fictive temperature), the temperature-independent (angular) reorientation of all molecules is expected to raise $\Delta \epsilon_{\mathrm{JG}}$. This is inconsistent with the observation in Fig. 2(B) that $\Delta \epsilon_{\mathrm{JG}}$ decreases irreversibly on heating initially, when structural relaxation dominates. Similar arguments have been used for examining the relative merits of the two relaxation mechanisms of the JohariGoldstein relaxation for other glasses earlier. ${ }^{9,11}$

\section{CONCLUSIONS}

The plot of the dielectric relaxation strength of the Johari-Goldstein relaxation against temperature resembles the plots of the volume, enthalpy and entropy against temperature in as much as it shows an elbow-shape in the glasssoftening range, and that this strength decreases on (spontancous) structural relaxation with time. Consequently, the relaxation strength is both time, and temperature dependent. The effect of structural relaxation dominates initially on heating and that of the temperature dominates ultimately, and hence a quenched glass shows a broad minimum in its plot of the relaxation strength in a temperature plane, resembling the plots of volume and enthalpy of a quenched glass measured during heating. Analysis of the data in the Arrhenius plot shows that the dielectric relaxation rate of the JohariGoldstein process would increase on structural relaxation. Arguments based upon the temperature dependence of the magnitude of the orientational polarization show that a mechanism in which only some D-sorbitol molecules confined to loosely packed regions in a disordered structure remain mobile seems preferable over one in which all molecules undergo a temperature-independent small-angle reorientation.

\section{ACKNOWLEDGMENTS}

G.P.J. wishes to thank Trinity College, Dublin, Ireland, for its hospitality during the period of this study. This research was supported by the Applied Research Program of Enterprise Ireland, and a Natural Sciences and Engineering Council of Canada's grant to G.P.J. for general research. J.K.V. thanks Intel Ireland for the award of Intel postgraduate studentship to G.P. for his research and training in dielectric materials.

${ }^{1}$ G. P. Johari, Ann. N.Y. Acad. Sci. 279, 117 (1976).

${ }^{2}$ D. A. Wasylyshyn, G. P. Johari, G. Salvetti, and E. Tombari, J. Phys.: Condens. Matter 9, 10521 (1997).

${ }^{3}$ D. A. Wasylyshyn, G. P. Johari, E. Tombari, and G. Salvetti, Chem. Phys. 223, 313 (1997).

${ }^{4}$ P. Lunkenheimer, U. Schneider, R. Brand, and A. Loidl, Contemp. Phys. 41, 15 (2000).

${ }^{5}$ See special Symposium volume issues of J. Non-Cryst. Solids 172 (1994); 173 (1994); 235-237, 393 (1998).

${ }^{6}$ U. Schneider, R. Brand, P. Lunkenheimer, and A. Loidl, Phys. Rev. Lett. 84, 5560 (2000)

${ }^{7}$ G. P. Johari, J. Chem. Phys. 58, 1766 (1973).

${ }^{8}$ R. Nozaki, D. Suzuki, S. Ozawa, and Y. Shiozaki, J. Non-Cryst. Solids 235-237, 393 (1998).

${ }^{9}$ G. P. Johari, J. Non-Cryst. Solids 307-310, 317 (2002).

${ }^{10}$ G. P. Johari, G. Power, and J. K. Vij, J. Chem. Phys. 116, 5908 (2002).

${ }^{11}$ G. P. Johari, G. Power, and J. K. Vij, J. Chem. Phys. 117, 1714 (2002).

${ }^{12}$ G. Power, G. P. Johari, and J. K. Vij, J. Chem. Phys. 116, 4192 (2002). 
${ }^{13}$ G. P. Johari, J. Non-Cryst. Solids 307-310, 114 (2002).

${ }^{14}$ G. P. Johari, Chem. Phys. 287, 273 (2003).

${ }^{15}$ J. M. Pelletier, B. van der Moortele, and I. R. Lu, in Proceeding of the 22nd Riso International Symposium on Materials Science: Science of Metastable and Non-Crystalline Alloys Structure, Properties and Modelling, edited by A. R. Dinesen, M. Eldrup, D. Juul Jensen, S. Linderoth, T. B. Pedersen, N. H. Prydes, A. Schroder Pedersen, and J. A. Wert (Riso National Laboratory, Roskilde, Denmark, 2001), p. 359.

${ }^{16}$ M. Naoki and K. Ujita, J. Chem. Phys. 99, 6971 (1993).

${ }^{17}$ O. E. Kalinovskaya and J. K. Vij, J. Chem. Phys. 112, 3262 (2000).

${ }^{18}$ G. P. Johari and M. Goldstein, J. Chem. Phys. 53, 2372 (1970).

${ }^{19}$ G. P. Johari, in Plastic Deformation of Amorphous and Semi-Crystalline Materials, edited by C. G'Sell and J. Perez (Les Edition de Physique, Les Ullis, France, 1983), p. 109.

${ }^{20}$ Gangasharan and S. S. Murthy, J. Phys. Chem. 99, 12349 (1995).

${ }^{21}$ S. Yagihara, M. Yamada, M. Asano, Y. Kanai, N. Shinyashiki, S. Mashimo, and K. L. Ngai, J. Non-Cryst. Solids 235-237, 412 (1998).

${ }^{22}$ H. Wagner and R. Richert, J. Phys. Chem. B 103, 4071 (1999).

${ }^{23}$ A. Kudlik, S. Benkhof, T. Blochowicz, C. Tschirwitz, and E. Rössler, J. Mol. Struct. 479, 201 (1999).

${ }^{24}$ S. Hensel-Bielowka, M. Paluch, J. Ziolo, and C. M. Roland, J. Phys. Chem. B 106, 12459 (2002).

${ }^{25}$ M. Naoki, K. Ujita, and S. Kashima, J. Phys. Chem. 97, 12356 (1993).

${ }^{26}$ N. B. Olsen, J. Non-Cryst. Solids 235-237, 399 (1998).

${ }^{27}$ H. Wagner and R. Richert, J. Non-Cryst. Solids 242, 19 (1998).

${ }^{28}$ A. Faivre, G. Niquet, M. Maglione, J. Fornazero, J. F. Lal, and L. David, Europhys. J. B 10, 277 (1999).

${ }^{29}$ T. Fujima, H. Frusawa, K. Ito, and R. Hayakawa, Jpn. J. Appl. Phys. 39, L744 (2000)

${ }^{30}$ E. Hempel, G. Hempel, A. Hansel, C. Schick, and E. Donth, J. Phys. Chem. B 104, 2460 (2000).

${ }^{31}$ R. Richert, Europhys. Lett. 54, 767 (2001).

${ }^{32}$ R. Nozaki, H. Zenitani, A. Minoguchi, and K. Kitai, J. Non-Cryst. Solids 307-310, 349 (2002).
${ }^{33}$ A. Minoguchi and R. Nozaki, J. Non-Cryst. Solids 307-310, 246 (2002).

${ }^{34}$ A. Döss, M. Paluch, H. Sillescu, and G. Hinze, Phys. Rev. Lett. 88, 095701 (2002).

${ }^{35}$ A. Döss, M. Paluch, H. Sillescu, and G. Hinze, J. Chem. Phys. 117, 6582 (2002).

${ }^{36}$ H. Yardimci and R. Leheny, Europhys. Lett. 62, 203 (2003).

${ }^{37}$ T. Fujima, H. Frusawa, and K. Ito, Phys. Rev. E 66, 031503 (2002).

${ }^{38}$ X.-H. Qiu and M. D. Ediger, J. Phys. Chem. B 107, 459 (2003).

${ }^{39}$ K. L. Ngai, R. W. Rendell, and D. J. Plazek, J. Chem. Phys. 94, 3018 (1991).

${ }^{40}$ J. Sztatisz, S. Gal, L. Fodor, and E. Pungor, J. Therm. Anal. 12, 351 (1977)

${ }^{41}$ C. Hansen, F. Stickel, T. Berger, R. Richert, and E. W. Fischer, J. Chem. Phys. 107, 1086 (1997).

${ }^{42}$ S. Havriliak, Jr. and S. Negami, J. Polym. Sci., Part C: Polym. Symp. 14, 99 (1966).

${ }^{43}$ R. H. Cole, J. Chem. Phys. 23, 493 (1955)

${ }^{44}$ K. Hofer and G. P. Johari, J. Chem. Phys. 95, 2020 (1991).

${ }^{45}$ K. L. Ngai, J. Phys.: Condens. Matter 15, S1107 (2003).

${ }^{46}$ G. W. Scherer, Relaxation in Glass and Composites (Wiley, New York, 1986).

${ }^{47}$ N. G. McCrum, B. E. Read, and G. Williams, Anelastic and Dielectric Effects in Polymeric Solids (Wiley, New York, 1967).

${ }^{48}$ J. Haddad and M. Goldstein, J. Non-Cryst. Solids 30, 1 (1978).

${ }^{49}$ G. P. Johari, Philos. Mag. B 46, 549 (1982).

${ }^{50}$ G. P. Johari and J. W. Goodby, J. Chem. Phys. 77, 5165 (1982).

${ }^{51}$ G. P. Johari, J. Chem. Phys. 77, 4619 (1982), and references therein.

${ }^{52}$ E. Tombari, S. Presto, G. Salvetti, and G. P. Johari, J. Chem. Phys. 117, 8436 (2002).

${ }^{53}$ G. P. Johari, J. Chem. Phys. 116, 8067 (2002).

${ }^{54}$ N. B. Olsen, T. Christensen, and J. C. Dyre, Phys. Rev. E 62, 4435 (2000).

${ }^{55}$ T. Fujima, H. Frusawa, K. Ito, and R. Hayakawa, Jpn. J. Appl. Phys. 38, L1046 (1999). 